\title{
Network synchronization: Optimal and Pessimal scale-free topologies
}

\author{
Luca Donetti \\ Departamento de Electrónica y Tecnología de Computadores and \\ Instituto de Física Teórica y Computacional Carlos I, Facultad de Ciencias, \\ Universidad de Granada, 18071 Granada, Spain
}

\author{
Pablo I. Hurtado and Miguel A. Muñoz \\ Departamento de Electromagnetismo y Física de la Materia and Instituto Carlos I de \\ Física Teórica y Computacional \\ Facultad de Ciencias, Universidad de Granada, 18071 Granada, Spain \\ E-mail: mamunoz@onsager.ugr.es
}

\begin{abstract}
.
By employing a recently introduced optimization algorithm we explicitely design optimally synchronizable (unweighted) networks for any given scale-free degree distribution. We explore how the optimization process affects degree-degree correlations and observe a generic tendency towards disassortativity. Still, we show that there is not a one-to-one correspondence between synchronizability and disassortativity. On the other hand, we study the nature of optimally unsynchronizable networks, that is, networks whose topology minimizes the range of stability of the synchronous state. The resulting "pessimal networks" turn out to have a highly assortative string-like structure. We also derive a rigorous lower bound for the Laplacian eigenvalue ratio controlling synchronizability, which helps understanding the impact of degree correlations on network synchronizability.
\end{abstract}

PACS numbers: 89.75.Hc,05.45.Xt,87.18.Sn

Keywords: Article preparation, IOP journals Submitted to: J. Phys. A: Math. Gen. 


\section{Introduction}

Synchronizability is one of the currently leading problems in the fast-growing field of Complex Networks [1]. A number of studies have been devoted to scrutinize which network topologies are more prone to sustain a stable globally-synchronized state of generic oscillators defined at each of its nodes [2, 3, 4, 5, 6, 7, 8, 9]. This question is of broad interest since many complex systems in fields ranging from physics, biology, computer science, or physiology, can be seen as networks of coupled oscillators, whose functionality depends crucially on the network ability to maintain a synchronous oscillation pattern. In addition, it has been shown that networks with good synchronizability are also "good" for (i) fast random walk spreading and therefore for efficient communication [8], (ii) searchability in the presence of congestion [10], (iii) robustness in the absence of privileged hubs [11], (iv) performance of neural networks [12], (v) generating consensus in social networks, etc. Another related and important problem that has received a lot of attention, but that we will not study here, is the dynamics towards synchronized states (see for example [13]).

In general terms, we can say that the degree of synchronizability is high when all the different nodes in a given network can "talk easily" to each other, or information

packets can travel efficiently from any starting node to any target one. It was first observed that adding some extra links to an otherwise regular lattice in such a way that a small-world topology [1] is generated, enhances synchronizability [2]. This was attributed to the fact that the node-to-node average distance diminishes as extra links are added. Afterwards, heterogeneity in the degree distribution was shown to hinder synchronization in networks of symmetrically coupled oscillators, leading to the so called "paradox of heterogeneity" as heterogeneity is known to reduce in average the node-tonode distance but still it suppresses synchronizability. The effect of other topological features as betweenness centrality, correlation in the degree distribution and clustering has been also analyzed. For example, it has been shown that the presence of weighted links (rather than uniform ones) and asymmetric couplings do enhance further the degree of synchronizability [5, 6], but here we focus on un-weighted and un-directed links.

Certainly, the main breakthrough was made by Barahona and Pecora [2] who, in a series of papers, established a criterion based on spectral theory to determine the stability of synchronized states under very general conditions. Their main contribution is to link graph spectral properties with network dynamical properties. In particular, they considered the Laplacian matrix, encoding the network topology, and showed that the degree of synchronizability (understood as the range of stability of the synchronous state) is controlled by the ratio between its largest eigenvalue $\left(\lambda_{N}\right)$ and the smallest non-trivial one $\left(\lambda_{2}\right)$, i.e. $Q=\lambda_{N} / \lambda_{2}$, where $N$ is the total number of nodes [14]. The smaller $Q$ the better the synchronizability.

Note that, as the range of variability of $\lambda_{N}$ is quite limited (it is directly related to the maximum connectivity [15, 16]), minimizing $Q$ is almost equivalent to maximizing the denominator $\lambda_{2}$ (i.e the spectral gap) when the degree distribution is kept fixed. 
It is worth noticing that, even if the eigenratio $Q$ can be related to (or bounded by) topological properties such as the ones cited above (average path length, betweenness centrality, etc.), none of these provides with a full characterization of a given network and therefore they are not useful to determine strict criteria for synchronizability [7]. Nevertheless, they can be very helpful as long as they give easy criteria to determine in a rough way synchronizability properties, without having to resort to lengthly eigenvalue calculations.

In a couple of recent papers, we tackled the problem of finding the optimally synchronizable topology, given a fixed number of nodes and edges linking them, and assuming symmetric and un-weighted links [8, 9]. The strategy we followed was to implement a simulated annealing algorithm [17] with a cost-function given by $Q$; starting with a random topology with $N$ nodes and $L$ links, random rewirings that decrease the value of $Q$ are accepted with larger probability than those increasing $Q$ (for more details see [8, 9]), until eventually a stationary (optimal or close to optimal) network is generated. Employing this optimization algorithm, we identified the family of "optimal network topologies" which we called entangled networks.

The main topological trait of entangled networks is the absence of bottlenecks and hubs; all sites are very much alike and the links form very intricate structures, which lead typically to (i) the absence of a well-defined community structure, (ii) poor modularity, and (iii) large shortest-loops. In this way, every single site is close to any other one owing to the existence of a very "democratic" or entangled structure in which properties such as site-to-site distance, betweenness, and minimum-loop-size are very homogeneously distributed (see [8, 9]).

Entangled networks were identified as Ramanujan graphs and they have been related to other interesting concepts in graph theory as expanders [18] and cage-graphs (see [9] and references therein). These are used profusely in computer science and are under current intense study in the mathematical literature. For example, expanders and Ramanujan graphs are very useful in the design of efficient communication networks, construction of error-correcting codes, or de-randomization of random algorithms [18, 9]. These applications greatly amplify the relevance of entangled networks in different contexts.

Despite of their mathematical beauty and excellent performance in network-design, entangled topologies are not easily found in biological, social, or any other "real-life" networks. An exception are some food-webs, for which topologies very similar to entangled ones have been reported [19]. As argued in [8, 9] the rarity in Nature of entangled networks comes from the fact that they emerge out of a global optimization process not easily fulfilled by means of any dynamical simple mechanism in growing networks where, usually, only local information is available.

Instead, real complex networks in very different contexts have been shown to exhibit, rather generically, scale-free degree distributions. These are much more heterogeneous than entangled topologies. Keeping this in mind, in this paper we explore the question of (global) optimization of synchronizability within the realm of scale-free 
networks with a fixed degree-distribution.

In particular, constraining our optimization algorithm to preserve a scale-free architecture, we are able to find the optimally synchronizable networks and study the emergence of non-trivial degree-degree correlations. This study is related to previous works by Sorrentino, di Bernardo and others [20], who argued that disassortative networks (in which nodes with similar degrees tend to be not connected among themselves) [21] are more synchronizable that assortative ones (where nodes with similar degrees tend to be connected).

Our study differs from previous ones in that (i) we derive a rigorous lowerbound for $Q$ in terms of a parameter measuring degree-degree correlations and (ii) we explicitly design optimal networks with a given degree-distribution and, by doing so, we verify that even if it is true that more disassortative networks typically exhibit better synchronizability, this is not always the case.

Finally, we also face the question of which are the pessimal networks for synchronization purposes. Actually, in some applications, synchronization (or consensus, or complete homogenization) are not desirable properties. This might be the case, for example, in neural networks for which global synchronization implies epileptic-like activity [22]. The question of how topology can hinder such states is both pertinent and relevant and also, it can give further insight on the key structural features of synchronization. With this goal in mind, we revert the optimization algorithm, and define an inverse optimization process just by maximizing $Q$ (rather than minimizing it), We analyze the topology of the resulting pessimal (or optimally un-synchronizable) networks.

\section{A rigorous upper bound for the spectral gap}

In this section we derive an upper bound for the spectral graph in terms of the correlation coefficient $r$. This coefficient was introduced by Newman in [21] to quantify the tendency of nodes with similar degrees, $k$, to be connected between themselves. In particular, calling $N$ the number of nodes and $L$ the total number of links $(L=N\langle k\rangle / 2)$ the correlation coefficient $r$ can be computed as (see [21] for more details):

$$
r=\frac{L^{-1} \sum_{i \sim j} k_{i} k_{j}-\left[L^{-1} \sum_{i \sim j} \frac{1}{2}\left(k_{i}+k_{j}\right)\right]^{2}}{L^{-1} \sum_{i \sim j} \frac{1}{2}\left(k_{i}^{2}+k_{j}^{2}\right)-\left[L^{-1} \sum_{i \sim j} \frac{1}{2}\left(k_{i}+k_{j}\right)\right]^{2}} .
$$

where $\sum_{i \sim j}$ stands for the sum over links (i.e. over all nodes $\mathrm{i}$ and $\mathrm{j}$ connected by a link; every link is counted only once). This parameter takes positive (negative) values for assortative (disassortative) configurations.

Defining the Laplacian matrix as $\mathcal{L}_{i j}=-1$ (0) if nodes $i$ and $j$ are connected (disconnected), and $\mathcal{L}_{i i}=k_{i}$, we can obtain an upper bound for $Q$ by recalling that the first non-trivial Laplacian eigenvalue $\lambda_{2}$ can be expressed as [15]:

$$
\lambda_{2}=2 N \min _{\mathbf{f} \in \Phi} \frac{\sum_{i \sim j}\left(f_{i}-f_{j}\right)^{2}}{\sum_{i} \sum_{j}\left(f_{i}-f_{j}\right)^{2}},
$$


where $\Phi$ is is the set of all possible non-constant vectors (in the space in which the Laplacian operator acts). Taking $\mathbf{f}=\left\{k_{i}, i=1 \ldots N\right\}$, which is one possible vector out of the set $\Phi$, we obtain:

$$
\lambda_{2} \leq 2 N \frac{\sum_{i \sim j}\left(k_{i}-k_{j}\right)^{2}}{\sum_{i} \sum_{j}\left(k_{i}-k_{j}\right)^{2}}=\frac{A}{\left\langle k^{2}\right\rangle-\langle k\rangle^{2}},
$$

where $A$ is defined as: $A=\frac{1}{N} \sum_{i \sim j}\left(k_{i}-k_{j}\right)^{2}$. A different selection of the vector would lead to a different inequality. The advantage of our choice is that the obtained bound can be related to the correlation coefficient $r$, even if it is not guaranteed that it provides a tight bound.

The different terms in the numerator and denominator of equation (1) can be written as

$$
\begin{aligned}
& \frac{1}{L} \sum_{i \sim j} \frac{1}{2}\left(k_{i}+k_{j}\right)=\frac{1}{N\langle k\rangle} \sum_{i} k_{i}^{2}=\frac{\left\langle k^{2}\right\rangle}{\langle k\rangle}, \\
& \frac{1}{L} \sum_{i \sim j} \frac{1}{2}\left(k_{i}^{2}+k_{j}^{2}\right)=\frac{1}{N\langle k\rangle} \sum_{i} k_{i}^{3}=\frac{\left\langle k^{3}\right\rangle}{\langle k\rangle} \\
& \frac{1}{L} \sum_{i \sim j} k_{i} k_{j}=\frac{1}{L} \sum_{i \sim j}\left(\frac{1}{2}\left(k_{i}^{2}+k_{j}^{2}\right)-\frac{1}{2}\left(k_{i}-k_{j}\right)^{2}\right)=\frac{\left\langle k^{3}\right\rangle-A}{\langle k\rangle} .
\end{aligned}
$$

By substituting these expressions in Eq. (11) and rearranging them we readily obtain:

$$
A=(1-r) \frac{\langle k\rangle\left\langle k^{3}\right\rangle-\left\langle k^{2}\right\rangle\left\langle k^{2}\right\rangle}{\langle k\rangle},
$$

and finally

$$
\lambda_{2} \leq(1-r) \frac{\langle k\rangle\left\langle k^{3}\right\rangle-\left\langle k^{2}\right\rangle\left\langle k^{2}\right\rangle}{\langle k\rangle\left(\left\langle k^{2}\right\rangle-\langle k\rangle^{2}\right)},
$$

which provides a rigorous upper bound for the spectral gap in terms of $r$. Observe that the more negative the value of $r$ (i.e. the more disassortative the network) the larger the upper bound for $\lambda_{2}$ and, therefore, $Q$ is allowed to take smaller values, and the corresponding network can be more synchronizable.

Summing up, the inequality Eq.(6) establishes that, as a rule of thumb, disassortative networks are more prone to have stable synchronized states than assortative ones, in agreement with previous results [20]. As a word of caution, let us underline that this does not imply that, given a fixed degree distribution, any disassortative network is better synchronizable than any assortative one, as we will illustrate in the following section.

A similar result to ours has been recently derived [20]. In particular, upper and lower bounds for $\lambda_{2}$ were obtained in terms of a parameter $\hat{r}$ quantifying the degreedegree correlation ( $\hat{r}$ is a simplified version of the more detailed one, $r$, defined by Eq.(1)). These upper and lower bounds were derived elaborating upon known bounds for the spectral gap in terms of the Cheeger constant [16, 15]. In order to obtain them, the authors implicitly assume that, for a fixed degree distribution, the Cheeger constant is an 


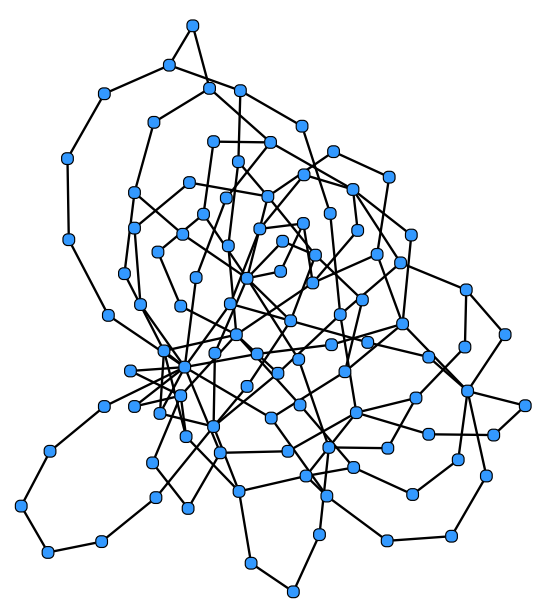

Figure 1. Uncorrelated scale-free network (with $\gamma=3, N=100$, and minimum connectivity $k=2$ ) from which the extremization processes leading to the networks in Fig. 2 is started.

uni-parametric function of $\hat{r}$. However, given a fixed $\hat{r}$, as this parameter does not specify completely the graph topology [7, different graphs with different Cheeger constants can be constructed. Therefore, the derivation of the bounds in [20] involves some type of mean-field-like approximation, while the upper bound here has been obtained in a rigorous way.

\section{Optimal and pessimal network design}

In this section we describe the optimization algorithm suitable for finding the network topology which extremizes the stability range of a global synchronous state in networks subject to a topological constraint: a fixed degree distribution. In particular, we apply this method to networks with scale-free topology and analyze the degree-degree correlations of the resulting (extremized) graphs.

The algorithm is a modified simulated-annealing [8] aimed at minimizing a cost function $\mathcal{F}(Q)$, where $Q=\lambda_{N} / \lambda_{2}$. A detailed description of the algorithm can be found either in [8] or in [9]. It yields networks for which the synchronizability is close to extremal (i.e. maximum or minimum), depending on the selected cost function $\mathcal{F}(Q)$. In particular, setting $\mathcal{F}(Q)=Q$ one gets networks with optimal synchronizability while choosing $\mathcal{F}(Q)=-Q$ the optimization procedure yields what we call pessimal networks.

The simulated-annealing rewiring process starts from networks generated using the configuration model [24]; in particular, it starts from connected networks with $N$ nodes and $L$ links, such that their degree distribution sample a power law $P(k) \sim k^{-\gamma}, \gamma>0$, with trivial (random) degree-degree correlation between neighboring nodes (see Fig,1). All the results in what follows correspond to $\gamma=3$. The graphs emerging out of the $Q$ and $-Q$ minimization processes, starting from the network in Fig. 1, are depicted in Fig,2. Naked eye inspection reveals the enormous differences between optimal and 

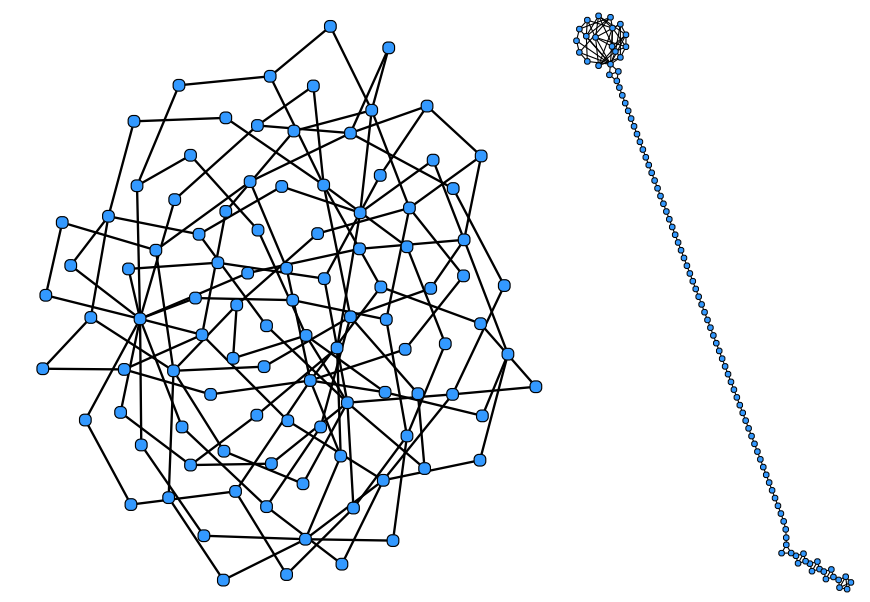

Figure 2. Left: Optimal scale-free network obtained from that in Fig. 1 via minimization of $Q$. Right: Pessimal scale-free network obtained from that in Fig. 1 via maximization of $Q$. The degree distributions of these two networks and the one in Fig. 1 are identical.

pessimal topologies. While the optimal ones resemble very much the very intricate and as-homogeneous-as-possible topology of entangled networks, pessimal topologies are as chain-like as possible, with two non-linear "heads" at both extremes, necessary to preserve the scale-free topology constraint. Note that large values of $Q$ imply small values of $\lambda_{2}$ and therefore, following the criterion for graph (bi)partitioning described for instance in [23], pessimal graphs have to be easily divisible into two parts by cutting an as-small-as-possible number of links. This is, indeed, achieved in an optimal way for linear (chain-like) topologies.

Let us remark, that different initial conditions with the same scale-free distribution, lead to outputs indistinguishable statistically from the ones in Fig.2, rendering robust the previous results.

To put these observations under a more quantitative basis, we measure degree correlations using (i) the average degree $\bar{k}_{n n}(k)$ of the neighbors of a node with degree $k$, and (ii) the correlation coefficient $r$ given by Eq.(1). Fig.3 shows $\bar{k}_{n n}(k)$, averaged over 10 different realizations, for initially uncorrelated networks (see, as an example, Fig. 11) as well as for the final optimal (Fig. 2, left) and pessimal (Fig. 2, right) networks. It reveals that optimally-synchronizable scale-free networks tend to display disassortative mixing (high-degree nodes tend to be connected with low-degree ones) while, on the contrary, pessimal scale-free networks tend to be assortative. This result agrees with the tendency predicted by the bound on the spectral gap in Eq. (6) as well as with previous results [20]. Actually, one could have anticipated these conclusions knowing that a network with good synchronization properties is also able to efficiently communicate any two nodes [8, 9]. In this sense, disassortative mixing, in which low connected nodes are preferentially linked to hubs which act as information distributors, seems most efficient.

On the other hand, pessimally synchronizable networks resulting from the 


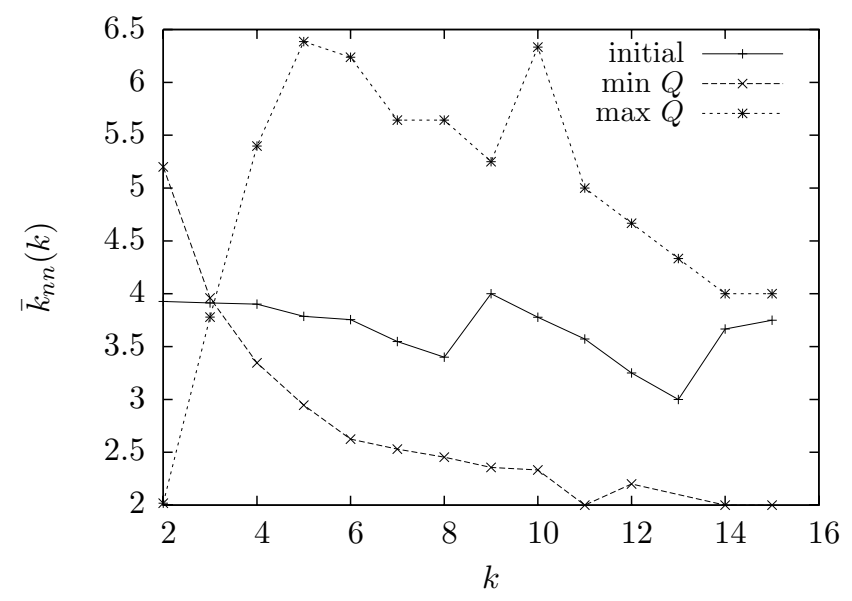

Figure 3. Ensemble average of $\bar{k}_{n n}(k)$ defined as the mean degree of the neighbors of a node with degree $k$, for (i) the initial uncorrelated scale-free networks with $\gamma=3$, (ii) the optimal scale-free networks resulting from the simulated annealing algorithm, and (iii) the pessimal scale-free networks obtained after maximization of $Q$. Results are averaged over an ensemble of 10 different networks of sizes $N=100$.

minimization of $-Q$ (or, equivalently, maximization of $Q$ ), tend to exhibit assortative mixing, i.e. $\bar{k}_{n n}(k)$ grows with $k$, at least up to a finite-size cutoff $k^{*}$, as shown in Fig. 3. The origin of such a cutoff is evident after realizing that the probability of having large hubs connected to other very large hubs must go to zero since the total number of links present in the system is finite. Obviously, the cutoff grows with system size and diverges asymptotically. The highly assortative chain-like topology of pessimal networks can be understood by the necessity of hampering the efficient communication between any two nodes in the system. This is achieved by maximizing the distance between any two hubs by interposing between them a linear chain of poorly-connected nodes.

Let us underline that the above observation on the effect of disassortative (assortative) mixing does not necessarily imply that maximizing disassortativity (assortativity) leads to optimal (pessimal) synchronizability. This is illustrated in Figs. 4,a-b (Figs. 4.d-e), which plot respectively the time evolution of the eigenratio $Q$ and the correlation coefficient $r$ during the optimization processes. The figure regarding optimization shows that the eigenratio $Q$ is not a monotonic function of $r$. This fact is made explicit in the insets to Figs. 4.a-b: the asymptotic minimun value of $Q$ does not correspond to the network for which $r$ is minimum (obtained in the example shown around $t=4 \times 10^{5}$ steps). This points out that, despite being a good indicator of synchronizability, disassortativity cannot be regarded as an unique topological measure of the stability of the synchronous state [7]. Moreover, further correlations apart from the observed assortativity/disassortativity are built up during the optimization process. In Fig. 4.c we plot the time evolution of the shortest-loop average length $\ell$, defined as the average over all nodes of the shortest loop passing through each node; it shows that $\ell$ grows during the minimization of $Q$, but again it exhibits a maximum before reaching 

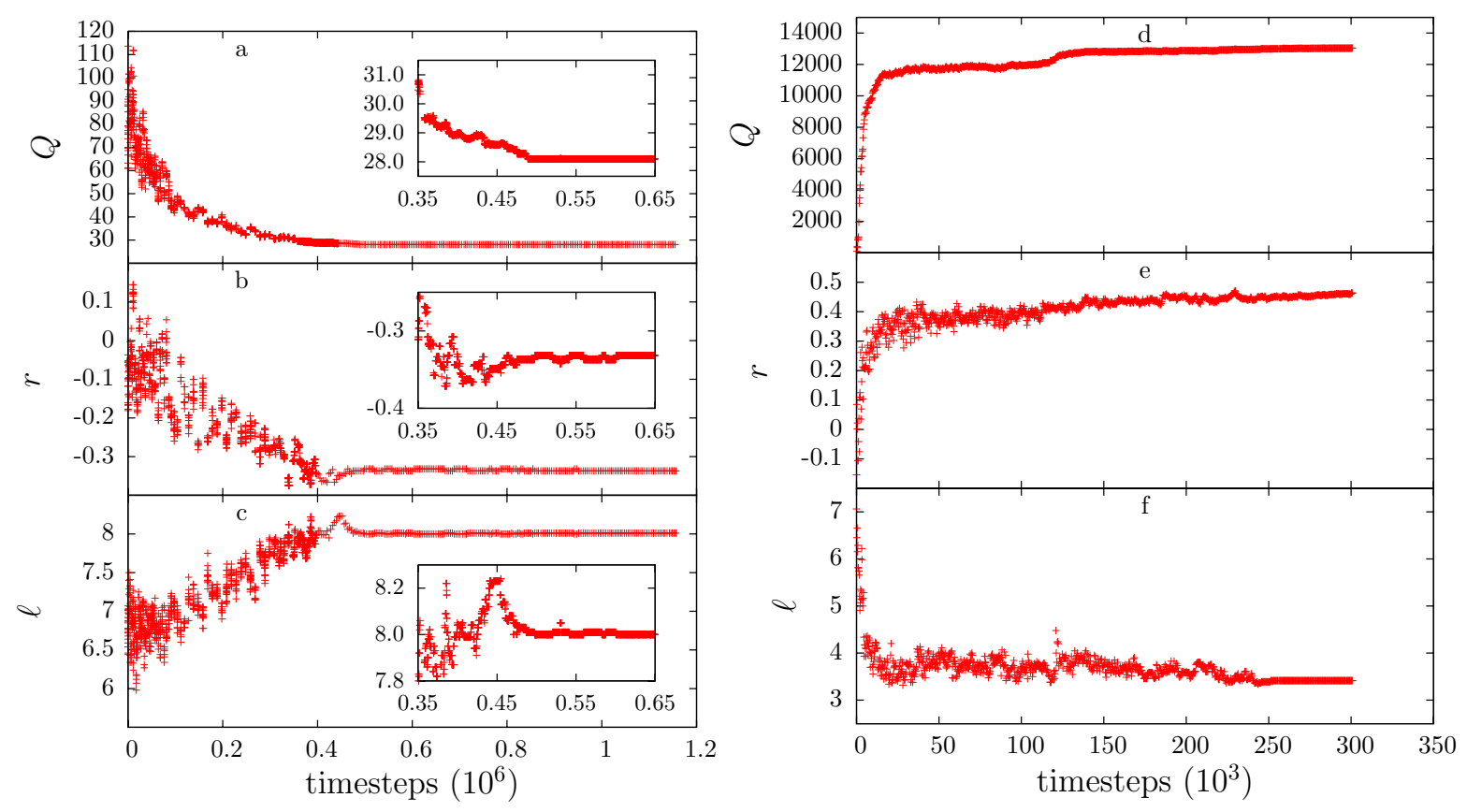

Figure 4. Time evolution of different quantities during the minimization (left) and maximization (right) procedure of a scale-free network with $\gamma=3, N=100$ nodes. (a) and (d) Eigenvalue ratio $Q$, (b) and (e) correlation coefficient $r$, (c) and (f) shortestloop average length $\ell$. The insets show a zoom of the corresponding curves. Note that $r$ (resp. $\ell$ ) exhibits a minimum (resp. maximum) during the algorithmic evolution which does not correspond to its optimal value.

its optimal-topology value. The tendency towards forming large loops for optimally synchronizable networks was reported before for entangled networks, where the smallest loops tend to be as large as possible [8, 9].

At this point we want to emphasize that the approach we have undertaken here is a constructive one, as opposed to that in [20], where different random networks with predefined degree distribution and correlations are explored to analyze how the externally-imposed assortative or disassortative correlation affects the eigenratio $Q$ of the resulting networks. Results here complement those in [20].

\section{Conclusions}

We have studied the problem of network synchronizability, which is directly related to many other important problems as efficient communication, searchability in the presence of congestion, many computer-science tasks, etc. While generically, the optimal networks for synchronizability, assuming un-weighted and un-directed links, are superhomogeneous, entangled topologies, in which all nodes look very much alike, in this work we have investigated the nature of optimal scale-free networks. The final goal is to analyze how are the degree-degree correlations of optimal scale-free networks.

For that, we have used the standard spectral approach consisting in relating the degree of synchronizability to the Laplacian matrix eigenratio $Q$. In a first part of this 
work, we have derived a rigorous lower bound for $Q$ in terms of the correlation coefficient $r$ (as defined by Eq.(1)), which is a measure of the degree-degree correlations. This lower bound turns out to be proportional $1 /(1-r)$, hence, showing that the more negative $r$ (i.e. the more disassortative the network) the smaller the lower bound and, therefore, the smaller values $Q$ is allowed to take, and the better the synchronizability of the resulting network.

In the second part, we have explicitly constructed optimal networks (with a fixed number of nodes, links, and a given scale-free degree distribution) by employing a recently introduced simulated-annealing algorithm [8, 9]. We find that optimal networks tend to be disassortative, as found already in previous studies [20], and in agreement with the expectations from the previously found lower bound. However, as there is not a one-to-one correspondence between $Q$ and the correlation coefficient $r$, more disassortative networks do not always synchronize better. Actually, we have illustrated how during the optimization process, at some point, the degree of assortativity increases (i.e. $r$ increases) as the network becomes more and more synchronizable. The emerging optimal networks exhibit also a tendency to have large loops and a rather intricate structure (as occurs for entangled networks).

Finally, we have reverted the optimization process and, by minimizing $-Q$, we have found what we call pessimally synchronizable networks. These topologies are characterized by a long string ended by two "heads" of nodes with degrees larger than 2 (required to preserve the scale-free degree distribution) and are, therefore, highly assortative. Contrarily to the case above, loops are very short. These topologies are the worst possible ones (compatible with the imposed scale-free degree distribution) if the task is to synchronize the network. But, on the contrary, they constitute the best choice if the goal is to avoid synchronization (or, equivalently, avoid communicability, searchability, homogenization), which might be important for some applications. For instance, in order to maximize the average time that a random infection (or random walk) takes to reach an arbitrary target node, this is the type of network to design.

We acknowledge financial support from the Spanish Ministerio de Educación y Ciencia (FIS2005-00791) and Junta de Andalucía (FQM-165).

\section{References}

[1] S. H. Strogatz, Nature 410, 268 (2001).

A. L. Barabási and R. Albert, Rev. Mod. Phys. 74, 47 (2002).

S. N. Dorogovtsev and J. F. F. Mendes, Evolution of Networks: From Biological Nets to the Internet and $W W W$, Oxford Univ. Press (2003).

M. E. J. Newman, SIAM Review 45, 167 (2003).

S. Boccaletti, V. Latora, Y. Moreno, M. Chavez, and D.-U. Hwang, Phys. Rep. 424, 175 (2006).

[2] M. Barahona and L. M. Pecora, Phys. Rev. Lett. 89, 054101 (2002).

See also, L. M. Pecora and T. L. Carroll, Phys. Rev. Lett. 64, 821 (1990); ibid, 80, 2109 (1998).

X. F. Wang and G. Chen, Int. J. Bifurcation Chaos Appl. Sci. Eng. 12, 187 (2002). 
[3] T. Nishikawa, A. E. Motter, Y.-C. Lai, and F. C. Hoppensteadt, Phys. Rev. Lett. 91, 014101 (2003).

[4] H. Hong, B. J. Kim, M. Y. Choi, and H. Park, Phys. Rev. E. 69, 067105 (2004). H. Hong, M. Y. Choi, and B. J. Kim, Phys. Rev. E 65, 026139 (2002); Phys. Rev. E 65, 047104 (2002).

[5] A. E. Motter, C. Zhou, and J. Kurths, Phys. Rev E 71, 016116 (2005); AIP Conference Proceedings 776, 201 (2005); Europhys. Lett. 69, 334 (2005).

T. Nishikawa and A. E. Motter, Phys. Rev. E 73, 065106 (2006).

A. E. Motter, New J. Phys. 9, 182 (2007).

[6] D.-U. Hwang, M. Chavez, A. Amann, and S. Boccaletti, Phys. Rev. Lett. 94, 138701 (2005).

M. Chavez, D.-U. Hwang, A. Amann, H. G. E. Hentschel, and S. Boccaletti, Phys. Rev. Lett. 94, 218701 (2005).

M. Chavez, D.-U. Hwang, and S. Boccaletti, Eur. Phys. J. Special Topics 146, 129 (2007).

[7] F. M. Atay, T. Biyikoglu, and J. Jost, Physica D 224, 35 (2006).

[8] L. Donetti, P. I. Hurtado and M. A. Muñoz, Phy. Rev. Lett. 95, 188701 (2005).

L. Donetti, P. I. Hurtado and M. A. Muñoz, Lect. Notes in Comp. Sci. 3993, 1075 (2006).

[9] L. Donetti, F. Neri, and M. A. Muñoz, J. Stat. Mech.: Theor. Exp. (2006) P08007.

[10] R. Guimera, A. Arenas, A. Diaz-Guilera, F. Vega-Redondo, and A. Cabrales, Phys. Rev. Lett. 89, 248701 (2002).

[11] V. Colizza, J. R. Banavar, A. Maritan, and A. Rinaldo, Phys. Rev. Lett. 92, 198701 (2004).

[12] J. J. Torres et al. Neurocomputing 58-60, 229 (2004). G. Grinstein and R. Linsker, Proc. Natl. Acad. Sci. USA, 102, 9948 (2005).

[13] J. Gómez-Gardeñes, Y. Moreno, and A. Arenas, Phys. Rev. Lett. 98, 034101 (2007).

J. Gómez-Gardeñes, Y Moreno, and A Arenas, Phys. Rev. E 75, 066106 (2007).

P. N. Graw and M. Menzinger, Phys. Rev. 72, 015101 (2005).

E. Oh, K. Rho, H. Hong, and B. Khang, Phys. Rev. E 72, 047101 (2005).

[14] Note that the Laplacian eigenvalues $\lambda_{i}$ satisfy $0=\lambda_{1} \leq \lambda_{2} \leq \ldots \leq \lambda_{N} \leq 2 k_{\max }$, where $k_{\max }$ is the largest degree in the graph [16, 15].

[15] B. Mohar, in Graph Theory, Combinatorics, and Applications, Vol 2, Ed. Y. Alavi, G. Chartrand, O. R. Oellermann, and A. J Schwenk, Wiley, New York, 1991. pp. 871.

[16] B. Bollobás, Extremal Graph Theory Academic Press, New York. 1978.

W. Tutte, Graph Theory As I Have Known It, Oxford U. Press, New York, (1998).

F. Chung, Spectral Graph Theory, Number 92 in CBMS Region Conference Series in Mathematics. Am. Math. Soc. 1997.

[17] N. Metropolis, A. W. Rosenbluth, M. N. Rosenbluth, A. H. Teller, and E. Teller, J. Chem. Phys. 211087 (1953).

T. J. P. Penna, Phys. Rev. E 51, R1 (1995).

[18] G. Davidoff, P. Sarnak and A. Valette, Elementary Number Theory, Group Theory and Ramanujan Graphs, London Math. Soc. Students Texts, 55, Cambridge (2003).

[19] E. Estrada, Phys. Rev. E 75, 016103 (2007); J. Theor. Biol. 244, 296 (2007).

[20] M. di Bernardo, F. Garofalo, and F. Sorrentino, Proceedings of the 44th IEEE Conference on Decision and Control, pp. 4616 (2005).

F. Sorrentino, M. di Bernardo, G. Huerta, Cúellar, and S. Boccaletti, Physica D 224, 123 (2006).

See also, B. Wang, H. Tang, T. Zhou, and Z. Xiu, arXiv:cond-mat/0512079.

[21] M.E.J. Newman, Phys. Rev. Lett. 89, 208701 (2002).

[22] L. Glass, Nature 410, 277 (2001).

[23] M. Girvan, M. E. J. Newman, Proc. Natl. Acad. Sci. USA, 99, 7821-7826 (2002). M. E. J. Newman, M. Girvan, Phys. Rev. E 69, 026113 (2004). See also, L. Donetti and M. A. Muñoz, J. Stat. Mech.: Theor. Exp. (2004) P10012; L. Donetti and M. A. Muñoz, in "Modeling Cooperative behavior in the social sciences", AIP Conf. Proc. 779, 104 (2005).

[24] See, M. Molloy and B. Reed, Comb. Prob. and Comput. 6, 161 (1995). M. Molloy and B. Reed, Comb. Prob. and Comput. 7, 295 (1998). 